\title{
Wer ist ... \\ Dirk Schadendorf?
}

Wie beginnen Sie Ihren Tag?

Schadendorf: Am liebsten in Ruhe mit genügend Zeit für ein ausgiebiges Frühstück und Zeitung dabei.

\section{Was treibt Sie an?}

Schadendorf: Die Lust, wissen zu wollen und (sichtbaren) Fortschritt zu machen. Mir macht es riesigen Spaß, Visionen zu entwickeln und vor allem auch an deren Realisierung zu arbeiten - für unsere Patienten, für die Sache, auch für das Team.

\section{Als Kind wollten Sie ...?}

Schadendorf: Möglichst die Dinge tun, die einem Spaß machen, ohne allzu viel Fremdbestimmung. Im Grunde hat sich dies bis heute erhalten... Sport, Bewegung und Outdoor-Aktivitäten waren für lange Jahre von großer Wichtigkeit in meiner Jugend. Dazu kamen ein sehr hoher Grad an Neugier und naturwissenschaftliches Interesse: Dies führte letztendlich dazu, dass ich ein Medizinstudium begann.

\section{Was beeindruckt Sie an anderen Menschen?}

Schadendorf: Menschen mit großer Offenheit, Freundlichkeit, Verlässlichkeit und Ehrlichkeit sind mir am sympathischsten. Am meisten beeindruckt mich, wenn dies gepaart ist mit einem gestalterischen Willen, der einem "größeren Ganzen" nützt. Mir imponiert besonders, wenn sich Menschen für Dinge leidenschaftlich einsetzen und begeistern.

\section{Worauf sind Sie besonders stolz?}

Schadendorf: Es gibt sehr viele Menschen und Dinge, auf die ich stolz bin. Dazu zählt die Familie, aber auch das Team rund um die Patientenversorgung sowie unsere Wissenschaftler und Netzwerke innerhalb und außerhalb von Essen.

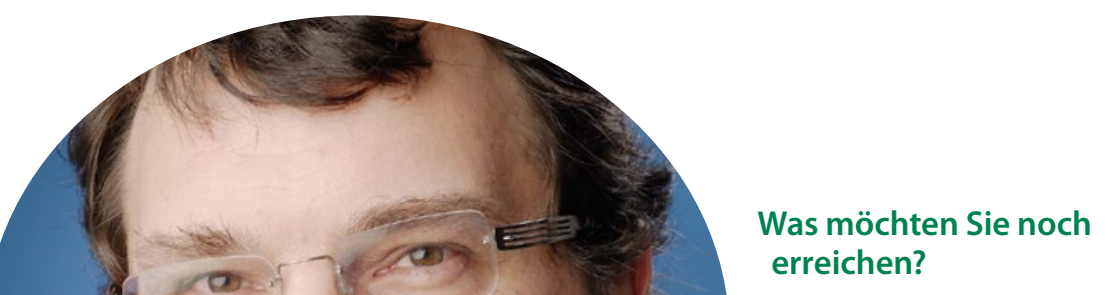

Schadendorf: Es gibt noch so viel zu tun und ich werde selbst nicht alles schaffen können. Zum einen würde ich gerne noch dazu beitragen, weitere therapeutische Verbesserungen bei der Behandlung von Hautkrebspatienten zu erzielen und so zumindest einem Teil dieser Patienten ein längerfristiges Überleben zu ermöglichen. Hierfür sind einerseits der Aufbau und die Vernetzung von Strukturen für Fort-, Aus- und Weiterbildung sowie Qualitätsinitiativen deutschlandweit notwendig, damit dieser Fortschritt auch möglichst vielen Menschen zugänglich wird; anderseits brauchen wir auch Forschungsstrukturen - von der Grundlagenbis zur Versorgungsforschung -, die immer wieder neue Impulse geben. Hierzu möchte ich in den nächsten Jahren auf verschiedenen Ebenen beitragen und die nächste Generation von Ärzten und Wissenschaftlern begeistern, weiter daran zu arbeiten. gefühl nahm er mit nach Berlin, u.a. für sechs Jahre in die Universitäts-Hautklinik des Virchow-Klinikums, FU Berlin.

Mit Frank Sinatras Zeilen im Ohr folgte Schadendorf dem Ruf der Universität Heidelberg auf eine C3-Professur. Dort leitete er von 1997 an für elf Jahre die Klinische Kooperationseinheit für Dermatoonkologie, eine Abteilung des Deutschen Krebsforschungszentrums, assoziiert mit der Hautklinik Mannheim. Seit Juli 2008 ist Schadendorf Direktor der Klinik für Dermatologie und des Westdeutschen Tumorzentrums am Universitätsklinikum Essen, außerdem ist er Vorsitzender der Arbeitsgemeinschaft Dermatologische Onkologie. 2010 erhielt Schadendorf zusammen mit Klaus Pantel und Ivan Đikić den Deutschen Krebspreis für die Erforschung des malignen Melanoms, insbesondere im Hinblick auf Prognose und therapeutische Resistenzen (klinischer Teil).

\section{Was war Ihre wichtigste} Entscheidung?

Schadendorf: Meine wichtigste Entscheidung war sicherlich, Medizin zu studieren und direkt nach meinem Studium ein Postdoc am Memorial Sloan-Kettering Cancer Institute zu machen.

\section{Wie halten Sie Balance in Ihrem Leben?}

Schadendorf: Das Loslassen der alltäglichen Herausforderungen und Gedanken gelingt mir am besten, wenn ich mir selbst Gutes tue, z. B. durch lange Spaziergänge.

\section{Wie beenden Sie Ihren Tag?}

Schadendorf: Mit einem Glas guten Rotweins, Musik und Kaminfeuer im Winter, um dann schnell und erschöpft einzuschlafen. 